\title{
Domain Decomposition Algorithm for Solving Contact of Elastic Bodies
}

\author{
Josef Daněk \\ University of West Bohemia, Univerzitní 22, 30614 Pilsen, Czech Republic \\ danek@kma.zcu.cz
}

\begin{abstract}
A nonoverlapping domain decomposition algorithm of Neumann-Neumann type for solving variational inequalities arising from the elliptic boundary value problems in two dimensions with unilateral boundary condition is presented. First, the linear auxiliary problem, where the inequality condition is replaced by the equality condition, is solved. In the second step, the solution of the auxiliary problem is used in a successive approximations method. In these solvers, a preconditioned conjugate gradient method with Neumann-Neumann preconditioner is used for solving the interface problems, while local problems within each subdomain are solved by direct solvers. A convergence of the iterative method and results of computational test are reported.
\end{abstract}

\section{Equilibrium of a system of bodies in contact}

We consider a system of elastic bodies decomposed into subdomains each of which occupies, in reference configuration, a domain $\Omega_{i}^{M}$ in $\mathbb{R}^{2}, i=1, \ldots, I_{M}$, $M=1, \ldots, \mathcal{J}$, with sufficiently smooth boundary $\partial \Omega_{i}^{M}$. Suppose that boundary $\bigcup_{M=1}^{\mathcal{J}} \partial \Omega^{M}$ consists of four disjoint parts $\Gamma_{u}, \Gamma_{\tau}, \Gamma_{c}$ and $\Gamma_{o}$ and that the displacements $u: \Gamma_{u} \rightarrow \mathbb{R}^{2}$ and forces $P: \Gamma_{\tau} \rightarrow \mathbb{R}^{2}$ are given. The part $\Gamma_{c}$ denote the part of boundary that may get into unilateral contact with some other subdomain and the part $\Gamma_{o}$ denote the part of boundary on that is prescribed the condition of the bilateral contact.

We shall look for the displacements that satisfy the conditions of equilibrium in the set $K=\left\{v \in V \mid v_{n}^{k}+v_{n}^{l} \leq 0\right.$ on $\left.\Gamma_{c}\right\}$ of all kinematically admissible displacements $v \in V, V=\left\{v \in \mathcal{H}^{1}(\Omega) \mid v=u_{0}\right.$ on $\Gamma_{u}, v_{n}=0$ on $\left.\Gamma_{o}\right\}, \mathcal{H}^{1}(\Omega)=$ $\left[H^{1}\left(\Omega_{1}^{1}\right)\right]^{2} \times \ldots \times\left[H^{1}\left(\Omega_{I_{\mathcal{J}}}^{\mathcal{J}}\right)\right]^{2}$ is standard Sobolev space. The displacement $u \in K$ of the system of bodies in equilibrium then minimizes the energy functional $\mathcal{L}(v)={ }_{2}^{1} a(v, v)-L(v)$ :

$$
\mathcal{L}(u) \leq \mathcal{L}(v) \text { for any } v \in K .
$$

Conditions that guarantee existence and uniqueness of the solution may be expressed in terms of coercivity of $\mathcal{L}$ and may be found, for example, in [1].

We define $\Gamma_{i}^{M}=\partial \Omega_{i}^{M} \backslash \partial \Omega^{M}$ and the interface $\Gamma=\bigcup_{M=1}^{\mathcal{J}} \bigcup_{i=1}^{I_{M}} \Gamma_{i}^{M}$. Let us introduce

$$
T^{M}=\left\{j \in\left\{1, \ldots, I_{M}\right\}: \quad \Gamma_{c} \cap \partial \Omega_{j}^{M}=\emptyset\right\}, \quad M=1, \ldots, \mathcal{J} .
$$


The number of a separate subset $\Gamma_{c}$ is $P_{c}$, i.e. $\Gamma_{c}=\bigcup_{j=1}^{P_{c}} \Gamma_{c j}$. Further, we denote

$$
\begin{gathered}
\Omega^{* j}=\left\{x \in \bigcup_{M=1}^{\mathcal{J}} \bigcup_{i=1}^{I_{M}} \Omega_{i}^{M}: \partial \Omega_{i}^{M} \cap \Gamma_{c j} \neq \emptyset\right\}, \quad j=1, \ldots, P_{c}, \\
\vartheta^{j}=\left\{[i, M]: \partial \Omega_{i}^{M} \cap \Gamma_{c j} \neq \emptyset\right\}, \quad j=1, \ldots, P_{c},
\end{gathered}
$$

i.e. $\Omega^{* j}=\bigcup_{[i, M] \in \vartheta^{j}} \Omega_{i}^{M}, j=1, \ldots, P_{c}$. We suppose that $\Gamma \cap \Gamma_{c}=\emptyset$ then $V_{\Gamma}=\left.\gamma K\right|_{\Gamma}=\left.\gamma V\right|_{\Gamma}$ for trace operator $\gamma:\left[H^{1}\left(\Omega_{i}^{M}\right)\right]^{2} \rightarrow\left[L^{2}\left(\partial \Omega_{i}^{M}\right)\right]^{2}$. We suppose that $\gamma^{-1}: V_{\Gamma} \rightarrow V$ is arbitrary linear inverse mapping for which

$$
\sum_{M=1}^{\mathcal{J}}\left(\gamma^{-1} \bar{v}^{M}\right)_{n}=0 \quad \forall \bar{v} \in V_{\Gamma} \quad \text { on } \Gamma_{c} .
$$

After denoting restrictions $\bar{R}_{i}^{M}: V_{\Gamma} \rightarrow \Gamma_{i}^{M}, L_{i}^{M}: L^{M} \rightarrow \Omega_{i}^{M}, a_{i}^{M}(.,):$. $a^{M}(.,.) \rightarrow \Omega_{i}^{M}, V\left(\Omega_{i}^{M}\right): V\left(\Omega^{M}\right) \rightarrow \Omega_{i}^{M}$ and introduction

$$
V^{0}\left(\Omega_{i}^{M}\right)=\left\{v \in V \mid v=0 \text { on }\left(\bigcup_{M=1}^{\mathcal{J}} \Omega^{M}\right) \backslash \Omega_{i}^{M}\right\},
$$

we can formulate the theorem 1 .

Theorem 1. A function $u \in K$ is the solution of the global problem (1) if and only if the function $u$ satisfies:

1.

$$
\sum_{M=1}^{\mathcal{J}} \sum_{i=1}^{I_{M}}\left(a_{i}^{M}\left(u_{i}^{M}(\bar{u}), \gamma^{-1} \bar{w}\right)-L_{i}^{M}\left(\gamma^{-1} \bar{w}\right)\right)=0 \quad \forall \bar{w} \in V_{\Gamma}, \bar{u} \in V_{\Gamma},
$$

for the trace $\bar{u}=\left.\gamma u\right|_{\Gamma}$ on the interface $\Gamma$.

2. Its rescriction $\left.u_{i}^{M}(\bar{u}) \equiv u\right|_{\Omega_{i}^{M}}$ satisfies following conditions:

a)

$$
\begin{gathered}
a_{i}^{M}\left(u_{i}^{M}(\bar{u}), \phi_{i}^{M}\right)=L_{i}^{M}\left(\phi_{i}^{M}\right) \quad \forall \phi_{i}^{M} \in V^{0}\left(\Omega_{i}^{M}\right), \\
u_{i}^{M}(\bar{u}) \in V\left(\Omega_{i}^{M}\right),\left.\gamma u_{i}^{M}(\bar{u})\right|_{\Gamma_{i}^{M}}=\bar{R}_{i}^{M} \bar{u},
\end{gathered}
$$

for $i \in T^{M}, M=1, \ldots, \mathcal{J}$,

b)

$$
\begin{aligned}
\sum_{[i, M] \in \vartheta^{j}} a_{i}^{M}\left(u_{i}^{M}(\bar{u}), \phi_{i}^{M}\right) & \geq \sum_{[i, M] \in \vartheta^{j}} L_{i}^{M}\left(\phi_{i}^{M}\right) \\
\forall \phi & \equiv\left(\phi_{i}^{M},[i, M] \in \vartheta^{j}\right), \phi_{i}^{M} \in V^{0}\left(\Omega_{i}^{M}\right)
\end{aligned}
$$

such that

$$
\begin{gathered}
u+\phi \in K \\
\left.\gamma u_{i}^{M}(\bar{u})\right|_{\Gamma_{i}^{M}}=\bar{R}_{i}^{M} \bar{u} \text { for }[i, M] \in \vartheta^{j},
\end{gathered}
$$

for $j=1, \ldots, P_{c}$. 


\section{The Schur complements}

We now want to write the interface problem (6) in operator form. For this purpose, we first introduce additional notation. We introduce the local trace spaces

$$
V_{i}^{M}=\left\{\left.\gamma v\right|_{\Gamma_{i}^{M}} \mid v \in K\right\}=\left\{\left.\gamma v\right|_{\Gamma_{i}^{M}} \mid v \in V\right\}
$$

and the extension $\operatorname{Tr}_{i M}^{-1}: V_{i}^{M} \rightarrow V\left(\Omega_{i}^{M}\right)$ defined by

$$
\begin{array}{r}
\left.\gamma\left(\operatorname{Tr}_{i M}^{-1} \bar{u}_{i}^{M}\right)\right|_{\Gamma_{i}^{M}}=\bar{u}_{i}^{M}, \\
a_{i}^{M}\left(\operatorname{Tr}_{i M}^{-1} \bar{u}_{i}^{M}, v_{i}^{M}\right)=0 \quad \forall v_{i}^{M} \in V^{0}\left(\Omega_{i}^{M}\right), \operatorname{Tr}_{i M}^{-1} \bar{u}_{i}^{M} \in V\left(\Omega_{i}^{M}\right), \\
\text { for } i \in T^{M}, M=1, \ldots, \mathcal{J} .
\end{array}
$$

For subdomains $\Omega^{* j}, j=1, \ldots, P_{c}$, we completed definition $T r_{i M}^{-1}$ with boundary condition

$$
\sum_{[i, M] \in \vartheta^{j}}\left(\operatorname{Tr}_{i M}^{-1} \bar{u}_{i}^{M}\right)_{n}=0 \text { on } \Gamma_{c j}, \text { for } j=1, \ldots, P_{c},
$$

i.e.

$$
\begin{aligned}
& \sum_{[i, M] \in \vartheta^{j}} a_{i}^{M}\left(\operatorname{Tr}_{i M}^{-1} \bar{u}_{i}^{M}, v_{i}^{M}\right)=0 \quad \forall\left(v_{i}^{M},[i, M] \in \vartheta^{j}\right): v_{i}^{M} \in V^{0}\left(\Omega_{i}^{M}\right), \\
& \text { so that } \sum_{[i, M] \in \vartheta^{j}}\left(v_{i}^{M}\right)_{n}=0 \text { on } \Gamma_{c j}, \quad j=1, \ldots, P_{c} .
\end{aligned}
$$

Definition 1. The local Schur complement, for $i \in T^{M}, M=1, \ldots, \mathcal{J}$, is operator $S_{i}^{M}: V_{i}^{M} \rightarrow\left(V_{i}^{M}\right)^{*}$ defined by

$$
\left\langle S_{i}^{M} \bar{u}_{i}^{M}, \bar{v}_{i}^{M}\right\rangle=a_{i}^{M}\left(\operatorname{Tr}_{i M}^{-1} \bar{u}_{i}^{M}, \operatorname{Tr}_{i M}^{-1} \bar{v}_{i}^{M}\right) \quad \forall \bar{u}_{i}^{M}, \bar{v}_{i}^{M} \in V_{i}^{M} .
$$

In matrix form, we have

$$
S_{i}^{M} \bar{U}_{i}^{M}=\left(\bar{A}_{i M}-B_{i M}^{T} \stackrel{\circ}{A}_{i M}^{-1} B_{i M}\right) \bar{U}_{i}^{M}
$$

where we decompose the degrees of freedom $U_{i}$ of $u_{i}$ into internal degrees of freedom $\stackrel{\circ}{U}_{i}^{M}$ and interface degrees of freedom $\bar{U}_{i}^{M}$ :

$$
U_{i}^{M}=\left[\stackrel{\circ}{U_{i}^{M}}, \bar{U}_{i}^{M}\right]^{T}
$$

With this decomposition, the matrix representation of $a_{i}^{M}(.,$.$) on H^{1}\left(\Omega_{i}^{M}\right)$ take the form

$$
A_{i M}=\left[\begin{array}{cc}
\stackrel{\circ}{A}_{i M} & B_{i M} \\
B_{i M}^{T} & \bar{A}_{i M}
\end{array}\right] .
$$


Definition 2. The combined local Schur complement, for subdomains $\Omega^{* j}, j=$ $1, \ldots, P_{c}$, is operator

$$
S_{* j}:\left(V_{i}^{M},[i, M] \in \vartheta^{j}\right) \rightarrow\left(V_{i}^{M},[i, M] \in \vartheta^{j}\right)^{*}, \quad j=1, \ldots, P_{c},
$$

defined by

$$
\begin{array}{r}
\left\langle S_{* j}\left(\bar{u}_{i}^{M},[i, M] \in \vartheta^{j}\right),\left(\bar{v}_{i}^{M},[i, M] \in \vartheta^{j}\right)\right\rangle=\sum_{[i, M] \in \vartheta^{j}} a_{i}^{M}\left(u_{i}^{M}\left(\bar{u}_{i}^{M}\right), \operatorname{Tr}_{i M}^{-1} \bar{v}_{i}^{M}\right) \\
\forall\left(\bar{v}_{i}^{M},[i, M] \in \vartheta^{j}\right) \in\left(V_{i}^{M},[i, M] \in \vartheta^{j}\right),
\end{array}
$$

where $u_{i}^{M}\left(\bar{u}_{i}^{M}\right)$ is the solution of the problem (8) and $\bar{R}_{i}^{M} \bar{u} \equiv \bar{u}_{i}^{M},[i, M] \in \vartheta^{j}$.

Lemma 1. The condition (6) for the function $\bar{u}$ on interface $\Gamma$ is equivalent to the condition (16):

$$
\begin{aligned}
& \sum_{M=1}^{\mathcal{J}} \sum_{i \in T^{M}}\left\langle S_{i}^{M} \bar{u}_{i}^{M}, \bar{w}_{i}^{M}\right\rangle+\sum_{j=1}^{P_{c}}\left\langle S_{* j}\left(\bar{u}_{i}^{M},[i, M] \in \vartheta^{j}\right),\left(\bar{w}_{i}^{M},[i, M] \in \vartheta^{j}\right)\right\rangle= \\
&=\sum_{M=1} \sum_{i \in I_{M}} L_{i}^{M}\left(\operatorname{Tr}_{i M}^{-1} \bar{w}_{i}^{M}\right), \quad \forall \bar{w} \in V_{\Gamma}, \text { where } \bar{w}_{i}^{M}=\bar{R}_{i}^{M} \bar{w}, \bar{u}_{i}^{M}=\bar{R}_{i}^{M} \bar{u}
\end{aligned}
$$

by using the local Schur complements.

We rewrite the condition (16) in the form

$$
S_{0} \bar{U}+S_{K O N} \bar{U}=F,
$$

where

$$
\begin{gathered}
S_{0}=\sum_{M=1}^{\mathcal{J}} \sum_{i \in T^{M}}\left(\bar{R}_{i}^{M}\right)^{T} S_{i}^{M} \bar{R}_{i}^{M}, \quad S_{K O N}=\sum_{j=1}^{P_{c}} \bar{R}_{* j}^{T} S_{* j} \bar{R}_{* j} \\
F=\sum_{M=1}^{\mathcal{J}} \sum_{i \in I_{M}}\left(\bar{R}_{i}^{M}\right)^{T}\left(T r_{i M}^{-1}\right)^{T} L_{i}^{M}
\end{gathered}
$$

and

$$
\bar{R}_{* j} \bar{u}=\left(\bar{R}_{i}^{M} \bar{u},[i, M] \in \vartheta^{j}\right)^{T}, \quad \bar{u} \in V_{\Gamma}, \quad \forall j=1, \ldots, P_{c} .
$$

By reason that operator $S_{K O N}$ is nonlinear, we solve the equation (17) successive aproximations method. We choose the solution of the auxiliary linear problem as an initial aproximation $\bar{U}^{0}$. In the auxiliary problem we replace the set $K$ by

$$
K^{0}=\left\{v \in V \mid \sum_{[i, M] \in \vartheta^{j}}\left(v_{i}^{M}\right)_{n}=0 \quad \text { on } \Gamma_{c j}\right\}
$$

and we obtain

$$
\begin{gathered}
u_{0}=\arg \min _{v \in K^{0}} \mathcal{L}(v), \\
\bar{U}^{0}=\left.\gamma u^{0}\right|_{\Gamma} .
\end{gathered}
$$

Now we come back to the equation (17) and we compute $\bar{U}^{k}$ as the solution of the linear problem

$$
S_{0} \bar{U}^{k}=F-S_{K O N} \bar{U}^{k-1}, \quad k=1,2, \ldots
$$




\section{The linearized problem}

We solve the variational equation

$$
u^{0} \in K^{0}, \quad D \mathcal{L}\left(u^{0}, v\right)=0 \quad \forall v \in K^{0} .
$$

For problem (19) we can describe the analogy of theorem 1 with one different in case $2 \mathrm{~b}$ ) where inequality is replaced by equality on $K^{0}$. For solution of this variational equality we define combined local Schur complement $S_{* j}^{0}, j=1, \ldots, P_{c}$ same as in definition 2 .

Definition 3. We define a global Schur complement:

$$
S=\sum_{j=1}^{P_{c}} \bar{R}_{* j}^{T} S_{* j}^{0} \bar{R}_{* j}+\sum_{M=1}^{\mathcal{J}} \sum_{i \in T^{M}}\left(\bar{R}_{i}^{M}\right)^{T} S_{i}^{M} \bar{R}_{i}^{M}
$$

and the condition (6) on the interface $\Gamma$ has form

$$
S \bar{U}=F
$$

in dual space $\left(V_{\Gamma}\right)^{*}$.

The equation (21) we solve by a conjugate gradient method with NeumannNeumann preconditioner. This method does not require the explicit construction of the local Schur complement matrix $S_{i}^{M}$ but does require an efficient preconditioner $\mathcal{M}^{-1}$. Its inverse $\left(S_{i}^{M}\right)^{-1}$, resp. $\left(S_{* j}^{0}\right)^{-1}$ simply consists in associating to the generalized derivative $g \in\left(V_{i}^{M}\right)^{*}$ the trace $\gamma \phi_{i}^{M}$ on $\Gamma_{i}^{M}$ of the solution $\phi_{i}^{M}$ of the corresponding Neumann problem.

Definition 4. We define an injection

$$
\begin{array}{ll}
D_{i}^{M}: & V_{i}^{M} \rightarrow V_{\Gamma}, \quad i \in T^{M}, \quad M=1, \ldots, \mathcal{J}, \\
D_{* j}: & \left(V_{i}^{M},[i, M] \in \vartheta^{j}\right) \rightarrow V_{\Gamma}, \quad D_{* j}=\left(D_{i}^{M},[i, M] \in \vartheta^{j}\right), \quad j=1, \ldots, P_{c},
\end{array}
$$

such that on each interface degree of freedom is

$$
D_{i}^{M} \bar{v}\left(P_{l}\right)=\bar{v}\left(P_{k}\right){ }_{\varrho_{T}}^{\varrho_{i}^{M}}, \quad i=1,2, \ldots I_{M}, M=1, \ldots, \mathcal{J},
$$

if the lth degree of freedom of $V_{\Gamma}$ corresponds to the $k$ th degree of freedom of $V_{i}^{M}$ and

$$
D_{i}^{M} \bar{v}\left(P_{l}\right)=0, \quad \text { if } n o t .
$$

Here $\varrho_{i}^{M}$ is a local measure of the stiffness of subdomain $\Omega_{i}^{M}$ (for example an average Young modulus on $\Omega_{i}^{M}$ ) and

$$
\varrho_{T}=\sum_{P_{l} \in \bar{\Omega}_{j}^{M}} \varrho_{j}^{M}
$$

is the sum of $\varrho_{j}^{M}$ on all subdomains na $\Omega_{j}^{M}$ containing $P_{l}$. 
The Neumann-Neumann precoditioner supposes that the solution of each local Neumann problem is uniquely defined, whereas rigid body motions are possible. This weakness can be fixed by replacing $\left(S_{i}^{M}\right)^{-1}$, resp. $\left(S_{* j}^{0}\right)^{-1}$ by a regularized inverse $\left(\tilde{S}_{i}^{M}\right)^{-1}$, resp. $\left(\tilde{S}_{* j}^{0}\right)^{-1}$. We introduce on each subdomain $\Omega_{i}^{M}$, resp. $\Omega^{* j}$ a small local coarse space $Z_{i}^{M}$, resp. $Z^{* j}$ containing all rigid body motion.

The general trick to upgrade the original preconditioner then consists in adding to the initial local contribution $\phi_{i}^{M}$, resp. $\phi_{j}$ a "bad" $z_{i}^{M} \in Z_{i}^{M}$, resp. $z_{j} \in Z^{* j}$ which is chosen in order to get the smallest difference $\left(\mathcal{M}^{-1}-S^{-1}\right)$.

We suppose that $L$ satisfies the invariance property

$$
\begin{gathered}
\left\langle L, D_{i}^{M} \gamma z_{i}^{M}\right\rangle=0 \quad \forall z_{i}^{M} \in Z_{i}^{M}, \quad i \in T^{M}, \quad M=1, \ldots, \mathcal{J}, \\
\left\langle L, D_{* j} \gamma z_{j}\right\rangle \equiv \sum_{[i, M] \in \vartheta^{j}}\left\langle L, D_{i}^{M} \gamma z_{i}^{M}\right\rangle=0 \quad \forall z_{j} \in Z^{* j}, \quad j=1, \ldots, P_{c} .
\end{gathered}
$$

We introduce a closed orthogonal complement space $Q\left(\Omega_{i}^{M}\right)$ of $Z_{i}^{M}$ in $V\left(\Omega_{i}^{M}\right)$ and a closed orthogonal complement space $Q\left(\Omega^{* j}\right)$ of $Z^{* j}$ in $\hat{V}_{j}$ where

$$
\hat{V}_{j}=\left\{\left(v_{i}^{M},[i, M] \in \vartheta^{j}\right) \mid v_{i}^{M} \in V\left(\Omega_{i}^{M}\right), \sum_{[i, M] \in \vartheta^{j}}\left(v_{i}^{M}\right)_{n}=0 \text { on } \Gamma_{c j}\right\} .
$$

Let then $\phi_{i}^{0 M} \in Q\left(\Omega_{i}^{M}\right)$ be the particular solution of the variational problem defined by

$$
a_{i}^{M}\left(\phi_{i}^{0 M}, v_{i}^{M}\right)=\left\langle L,\left.D_{i}^{M}\left(\gamma v_{i}^{M}\right)\right|_{\Gamma_{i}^{M}}\right\rangle \quad \forall v_{i}^{M} \in V\left(\Omega_{i}^{M}\right)
$$

and $\phi_{* j}^{0}=\left(\phi_{i}^{0 M},[i, M] \in \vartheta^{j}\right) \in Q\left(\Omega^{* j}\right)$ be the particular solution of the variational problem defined by

$$
\sum_{[i, M] \in \vartheta^{j}} a_{i}^{M}\left(\phi_{i}^{0 M}, v_{i}^{M}\right)=\sum_{[i, M] \in \vartheta^{j}}\left\langle L,\left.D_{i}^{M}\left(\gamma v_{i}^{M}\right)\right|_{\Gamma_{i}^{M}}\right\rangle \quad \forall v_{j} \in \hat{V}_{j} .
$$

Equations (28), (29) are well posed varitional problems set on $Q\left(\Omega_{i}^{M}\right), Q\left(\Omega^{* j}\right)$.

Definition 5. We define Neumann-Neumann preconditioner $\mathcal{M}^{-1}\left(z^{0}\right)$ by

$$
\mathcal{M}^{-1}\left(z^{0}\right) L=\left.\sum_{M=1}^{\mathcal{J}} \sum_{i=1}^{I_{M}} D_{i}^{M} \gamma\left(\phi_{i}^{0 M}+z_{i}^{0 M}\right)\right|_{\Gamma_{i}^{M}},
$$

with the solution $z_{i}^{0 M}$ of the minimization problem

$$
\begin{gathered}
z^{0}=\arg \min _{z \in \Pi Z}\left\langle S\left(\mathcal{M}^{-1}(z)-S^{-1}\right) \underset{J(z)}{L}\left(\mathcal{M}^{-1}(z)-S^{-1}\right) L\right\rangle, \\
\Pi Z \equiv\left(\bigotimes_{i \in T^{M}, M=1, \ldots, \mathcal{J}}\left(Z_{i}^{M}\right)\right) \times\left(\bigotimes_{j=1, \ldots, P_{c}}\left(Z^{* j}\right)\right) .
\end{gathered}
$$




\section{Successive approximations method}

Now we solve, by the successive approximations method, the equation (18). We must effectively compute the solution $\bar{U}^{k}$ of the linear problem

$$
S_{0} \bar{U}^{k}=b^{k},
$$

with

$$
\begin{gathered}
S_{0}=\sum_{M=1}^{\mathcal{J}} \sum_{i \in T^{M}}\left(\bar{R}_{i}^{M}\right)^{T} S_{i}^{M} \bar{R}_{i}^{M}, \quad b^{k}=F-S_{K O N} \bar{U}^{k-1}, \\
F=\sum_{M=1}^{\mathcal{J}} \sum_{i \in I_{M}}\left(\bar{R}_{i}^{M}\right)^{T}\left(\operatorname{Tr}_{i M}^{-1}\right)^{T} L_{i}^{M}, \quad S_{K O N}=\sum_{j=1}^{P_{c}} \bar{R}_{* j}^{T} S_{* j} \bar{R}_{* j} .
\end{gathered}
$$

The equation (32) we solve by a preconditioned conjugate gradient method.

Definition 6. We define an injection

$$
D_{i}^{M}: \quad V_{i}^{M} \rightarrow V_{\Gamma}, \quad i \in T^{M}, \quad M=1, \ldots, \mathcal{J},
$$

such that on each interface degree of freedom is

$$
\begin{array}{r}
D_{i}^{M} \bar{v}\left(P_{l}\right)=\bar{v}\left(P_{k}\right), \quad \text { if } P_{k} \in \Gamma_{i}^{M} \subset \partial \Omega^{* j} \text { for any } j \in\left\{1, \ldots, P_{c}\right\}, \\
D_{i}^{M} \bar{v}\left(P_{l}\right)=\bar{v}\left(P_{k}\right)_{\varrho_{T}}^{\varrho_{T}^{M}}, \quad \text { for } P_{k} \in \Gamma_{i}^{M} \not \subset \partial \Omega^{* j} \quad \forall j=1, \ldots, P_{c},
\end{array}
$$

if the lth degree of freedom of $V_{\Gamma}$ corresponds to the $k$ th degree of freedom of $V_{i}^{M}$ and

$$
D_{i}^{M} \bar{v}\left(P_{l}\right)=0, \quad \text { if not. }
$$

Let $\phi_{i}^{0 M} \in Q\left(\Omega_{i}^{M}\right)$ be the particular solution of the variational problem defined by (28). Similarly to the linearized problem we define a preconditioner.

Definition 7. We define Neumann-Neumann preconditioner $\mathcal{M}_{o}^{-1}$ by

$$
\mathcal{M}_{o}^{-1}\left(z^{0}\right) L=\left.\sum_{M=1}^{\mathcal{J}} \sum_{i \in T^{M}} D_{i}^{M} \gamma\left(\phi_{i}^{0 M}+z_{i}^{0 M}\right)\right|_{\Gamma_{i}^{M}},
$$

with the solution $z_{i}^{0 M}$ of the minimization problem

$$
\begin{gathered}
z^{0}=\arg \min _{z \in \Pi_{o} Z}\left\langle S_{0}\left(\mathcal{M}_{o}^{-1}(z)-S_{0}^{-1}\right) L,\left(\mathcal{M}_{o}^{-1}(z)-S_{0}^{-1}\right) L\right\rangle, \\
\Pi_{o} Z \equiv \bigotimes_{i \in T^{M}, M=1, \ldots, \mathcal{J}}\left(Z_{i}^{M}\right) .
\end{gathered}
$$


We introduce the coarse trace space

$$
V_{o H}=\sum_{M=1}^{\mathcal{J}} \sum_{i \in T^{M}} D_{i}^{M} \gamma Z_{i}^{M}
$$

a set $V_{o H}^{\perp} \subset\left(V_{\Gamma}\right)^{*}$ given by

$$
L \in V_{o H}^{\perp} \Leftrightarrow\langle L, z\rangle=0 \quad \forall z \in V_{o H} .
$$

A convergence theorem requires to introduce some definitions. Let $\Theta$ be an ortogonal complement of $V_{o H}$ in $V_{\Gamma}$. We introduce seminorms

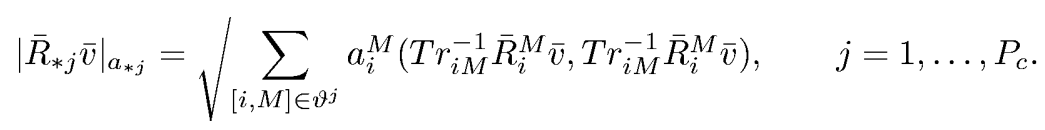

Lemma 2. The expression

$$
\|\bar{u}\|_{Q}^{2}=\left\langle S_{0} \bar{u}, \bar{u}\right\rangle
$$

is a norm on $\Theta$ where

$$
Q=\bigotimes_{i, M: i \in T^{M} ; M=1, \ldots, \mathcal{J}} Q\left(\Omega_{i}^{M}\right) .
$$

Definition 8. Let $\mathcal{T}: \Theta \rightarrow \Theta$ be a mapping defined by

$$
\left\langle S_{0}(\mathcal{T} \bar{y}), \bar{v}\right\rangle=\left\langle F-S_{K O N}(\bar{y}), \bar{v}\right\rangle \quad \forall \bar{v} \in \Theta .
$$

Theorem 2. Assume that there exists a constant $\lambda<\underset{\sqrt{ } 2 P_{c}}{1}$ such that the following condition hold:

$$
\left|\bar{R}_{* j} \bar{u}\right|_{a_{* j}} \leq \lambda\|\bar{u}\|_{Q}, \quad \forall \bar{u} \in \Theta, \quad \forall j \in\left\{1, \ldots, P_{c}\right\} .
$$

Then the mapping $\mathcal{T}$ is the contraction on $\Theta$. If $\bar{U}^{0} \in \Theta$ then the sequence of the iterations $\bar{U}^{k}$, computed by (32), are convergent and the limit is a fixed point $\bar{U}$ of the mapping $\mathcal{T}$. The following error estimate holds

$$
\left\|\bar{U}^{k}-\bar{U}\right\|_{Q} \leq \begin{gathered}
\left(2 \lambda^{2} P_{c}\right)^{k} \\
1-2 \lambda^{2} P_{c}
\end{gathered}\left\|\bar{U}^{0}-\mathcal{T} \bar{U}^{0}\right\|_{Q}
$$

\section{$5 \quad$ Numerical experiments}

In this section, we illustrate the practical behavior of our algorithm on solution of the geomechanical model problem describing loaded tunnel which is crossing by the deep fault and based on the geomechanical theory and models having connection with radioactive waste repositories (see [3]). The introduced algorithm has been implemented in MPI version 1.2.0 by using FORTRAN 77 compiler. A geometry of the problem is in Fig. 1. 
Material parameters: 2 regions with Young's modulus $E=0.52^{10}[\mathrm{~Pa}]$ and Poisson's ratio $\nu=0,18$.

Boundary conditions: Prescribed displacement $\left(-2,5 \times 10^{-2}, 0\right)[\mathrm{m}]$ on $3-4$. Pressure $0,5 \times 10^{7}[\mathrm{~Pa}]$ on 1-4. Bilateral contact boundary: 1-2 and 2-3. Unilateral contact boundary: 5-6 and 7-8.

Discretization statistics: 12 subdomains, 5501 nodes, 9676 elements, 10428 unknowns, 89 unilateral contact conditions, 466 interface elements.

Convergence statistics: 19 iterations of the PCG algorithm for the auxiliary problem 14 iterations of the successive approximations method, total 38 iterations of the PCG algorithm for the original problem.

Fig. 2 represents detail of deformations and Fig. 3 demonstrates detail of principal stresses in model problem.

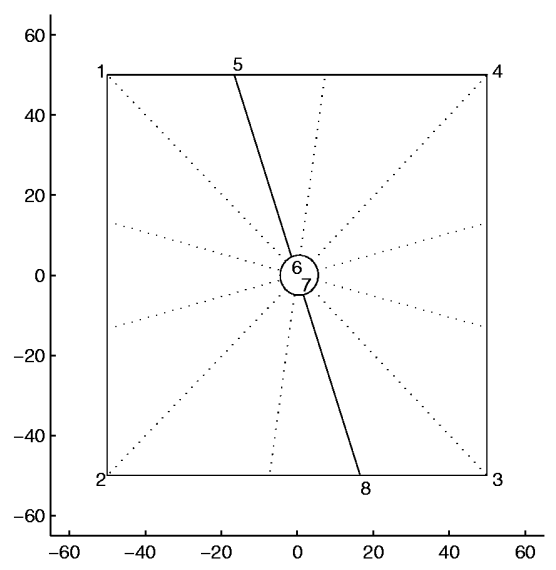

Fig. 1. A geometry of the problem

\section{References}

1. Hlaváček, I., Haslinger, J., Nečas, J., Lovíšek, J.: Solution of Variational Inequalities in Mechanics. Appl. Math. Sci. 66, Springer-Verlag, New York, 1988

2. Hlaváček, I.: Domain Decomposition Applied to a Unilateral Contact of Elastic Bodies. Technical report V-785 ICS AS CR, Prague 1999

3. Nedoma, J.: Numerical Modelling in Applied Geodynamics. Wiley, Chichester, 1998

4. Le Tallec, P.: Domain Decomposition Methods in Computational Mechanics. Computational Mechanics Advances 1 (pp. 121 - 220), North-Holland, 1994

5. Le Tallec, P., Vidrascu, M.: Generalized Neumann-Neumann Preconditioners for Iterative Substructuring. Proceedings of the 9th International Conference on Domain Decomposition Method, pp. 413-425, 1998

6. Daněk, J.: A solving unilateral contact problems with small range of contact in $2 D$ by using parallel computers. Ph.D. Thesis, UWB Pilsen, 2001.

7. Daněk, J.: Domain decomposition method for contact problems with small range contact. J. Mathematics and Computers in Simulation, Proceedings of the conference Modelling 2001, UWB Pilsen, 2001. 


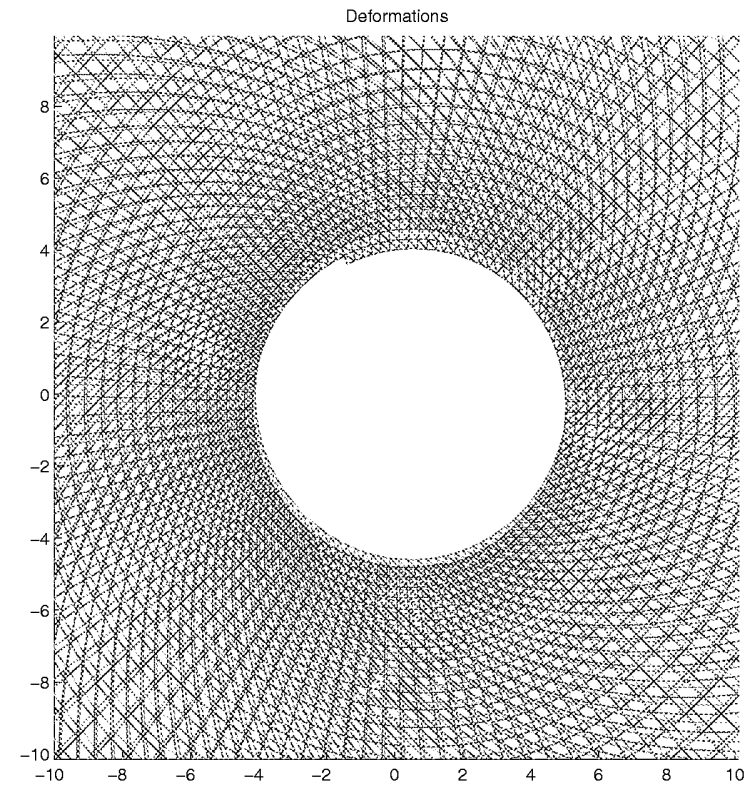

Fig. 2. A detail of deformations in model problem (enlarge factor=10)

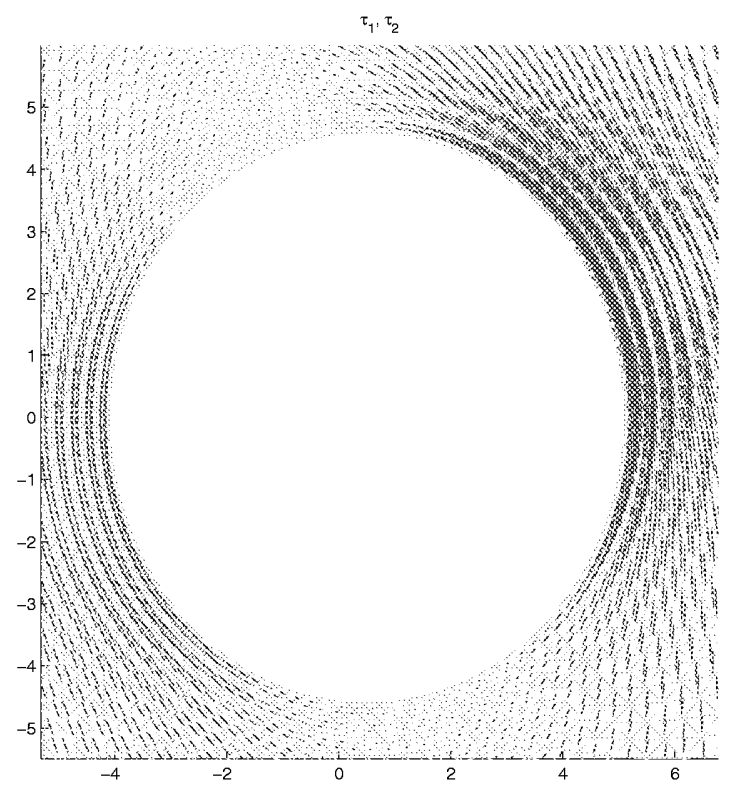

Fig. 3. A detail of principal stresses in neighbourhood of the tunnel. Figure show that maximal pressure is along right-hand side of the tunnel. 